\title{
Concurrent Expression of VEGF-C and Neuropilin-2 Is Correlated with Poor Prognosis in Glioblastoma
}

\author{
Haiqing Zhao, ${ }^{1, *}$ Chunfeng Hou, ${ }^{1, *}$ Aiwu Hou ${ }^{1}$ and Dongyuan $\mathrm{Zhu}^{2}$ \\ ${ }^{1}$ Department of Neurology, Yidu Central Hospital of Weifang City, Weifang, Shandong, China \\ ${ }^{2}$ Department of Medical Oncology, Shandong Cancer Hospital and Institute, Shandong Academy of Medical \\ Sciences, Jinan, Shandong, China
}

\begin{abstract}
Vascular endothelial growth factor-C (VEGF-C) is a secreted growth factor involved in many oncogenic processes, and neuropilin-2 (NRP2) is essential for neuronal guidance as a well-acknowledged co-receptor of VEGF receptors. The overexpression of NRP2 has been reported in many types of cancers, but the significance of NRP2 in glioblastoma is not elucidated. To investigate the clinical significance of VEGF-C and NRP2 in glioblastoma, we detected their expression in 86 cases of glioblastoma with immunohistochemistry and categorized our cohort into high- and low-expression groups according to the immunohistochemistry score, which was the product of the score of staining intensity multiplied by the score reflecting the percentage of positive cells. The proportion of glioblastoma with high VEGF-C expression was $34.9 \%$ (30/86), and that with high NRP2 expression was $37.2 \%(32 / 86)$. The proportion of glioblastoma with high expression of both VEGF-C and NRP2 was 15.1\% (13/86). Moreover, the proportion of cases with high VEGF-C and low NRP2 was $19.7 \%(17 / 86)$, while the proportion of cases with low VEGF-C and high NRP2 was $22.1 \%(19 / 86)$. The high expression of both VEGF-C and NRP2 was associated with poorer survival rate $(P=0.023)$, and can be identified as an independent prognostic factor in glioblastoma $(P=0.030)$. Moreover, cases with low NRP2 expression are easier for complete tumor resection $(P=0.038)$. In conclusion, the concurrent high expression of VEGF-C and NRP2 is predictive of the unfavorable prognosis in glioblastoma, indicating that the VEGF-C-NRP2 signaling pathway is a potential and promising drug target in glioblastoma therapy.
\end{abstract}

Keywords: biomarker; glioblastoma; neuropilin-2; prognosis; vascular endothelial growth factor-C Tohoku J. Exp. Med., 2016 February, 238 (2), 85-91. (C) 2016 Tohoku University Medical Press

\section{Introduction}

The neuropilins (NRPs) are one class of vascular endothelial growth factor (VEGF) receptors, including neuropilin-1 (NRP1) and neuropilin-2 (NRP2), which were identified initially as neuronal receptors for semaphorins and essential for both neuronal guidance and cardiovascular development (Prud'homme and Glinka 2012; Plein et al. 2014). NRPs were reported to interact with VEGF receptors (VEGFR1 and VEGFR2) and other receptors (Sulpice et al. 2008). Importantly, NRP1 was demonstrated to promote the viability of stem-like cell and tumor growth of glioma, and NRP1 interference could inhibit glioma growth in vivo and in vitro (Nasarre et al. 2010; Li et al. 2011; Hamerlik et al. 2012). Compared with the breakthrough of NRP1 research in glioma, the role of NRP2 has not been well elucidated, except for the study proving that NRP2 could promote the growth and clonogenicity of glioblastoma multiforme cell lines (Epis et al. 2014). In addition,
NRP2 was overexpressed and associated with poor prognosis in several types of human tumors (Caunt et al. 2008; Goel et al. 2012).

Glioma is the most common malignancy of central nerve system in adults, and glioblastoma is the most malignant and lethal glioma (grade IV according to the WHO classification). The incidence of glioblastoma is 3-6 per 100,000 individuals, which accounts for approximately $50 \%-60 \%$ of all astrocytic gliomas and $12 \%-15 \%$ of all intracranial neoplasms (Wrensch et al. 2002; Louis et al. 2007; Wen and Kesari 2008). The standard first-line of glioblastoma treatment is usually surgery, followed by radiation therapy or combined radiation and chemotherapy. The patients with glioblastoma are rarely curative and most patients would suffer tumor recurrence (Nicolaidis 2015). The 5-year survival rate of glioblastoma is just $3 \%$ and the median survival time is just 15 months despite the developing methods of resection and adjuvant therapies including maximal safe resection followed by radiotherapy in combi-

Received August 24, 2015; revised and accepted December 15, 2015. Published online January 9, 2016; doi: 10.1620/tjem.238.85.

*These two authors contributed equally to this work.

Correspondence: Dongyuan Zhu, M.D., Department of Medical Oncology, Shandong Cancer Hospital and Institute, Shandong Academy of Medical Sciences, 440\# Jiyan Road, Jinan, Shandong 250117, China.

e-mail: dyzdyzshandong@163.com 
nation with temozolomide (Meng et al. 2015). It is generally accepted that new breakthroughs of glioblastoma therapies depend on establishing patient's genotype and proteomic profile of biomarkers. However, although significant progresses have been made in molecular biology of glioblastoma, there still exists an urgent need for identification of new biomarkers to predict new approaches for treating glioblastoma.

In the present study, we investigated 190 patients with glioblastoma and enrolled 86 cases into the validation cohort. Furthermore, we detected the expression of VEGF-C and NRP2 in glioblastoma tissues with immunohistochemistry method and in glioblastoma cell lines with immunoblotting analysis. Moreover, we analyzed the correlation between VEGF-C and NRP2 expression and the clinicopathologic factors of glioblastoma with Chi-square test, and then evaluated the prognostic value of VEGF-C and NRP2 expression with univariate and multivariate analysis.

\section{Patients and Methods}

\section{Patients and follow-up}

A total of 190 cases of patients who underwent macroscopic total or near-total tumor resection were diagnosed as glioblastoma and underwent the surgery at three clinical centers (Central Hospital of Jinan City, Yidu Central Hospital of Weifang City, People's Hospital of Qingzhou City) from 2003 to 2014, which comprised the primary cohort. The validation cohort contained 86 cases selected from the primary cohort following the criteria as: (1) available follow-up and samples; (2) post-operational survival time was more than 1 month. This study was reviewed and approved by the committee on ethics of all the three clinical centers. The samples were obtained from the Pathology department with prior patient consents. The overall survival time was identified as the time from the operation to the date of death or censored at the date of the last follow-up examination. All the patients in validation cohort underwent the evaluation of Karnofsky Performance Scale (KPS).

\section{Cell culture and reagents}

Human glioma cell line U251MG and human glioblastoma cell lines U118MG and U87MG were all purchased from Cell Bank of the Chinese Academy of Sciences (Shanghai, China) and cultured in DMEM containing 10\% fetal bovine serum (FBS) supplemented with streptomycin $(100 \mu \mathrm{g} / \mathrm{ml})$ and penicillin $(100 \mathrm{U} / \mathrm{ml})$. The antibodies without special instructions were all purchased as follows: anti-NRP2 (sc-13117, Santa Cruz Biotechnology, Santa Cruz, CA, USA); antiVEGF-C (AP2042d, ABGENT, San Diego, CA, USA) ; and anti- $\beta$ actin (sc-47778, Santa Cruz Biotechnology, Santa Cruz, CA, USA) .

\section{Immunohistochemistry (IHC) and evaluation}

Expression of VEGF-C and NRP2 was detected by the streptavidin-biotin immunoperoxidase assay. Briefly, $3 \%$ hydrogen peroxide was used to soak the slides for endogenous peroxidase inactivation. After that, citrate buffer $(\mathrm{pH}=6.0)$ was used for immunoreactivity enhancement and $5 \%$ bovine serum albumin for unspecific binding exclusion. The samples were then incubated with primary antibody at 1:50 to $1: 100$ dilution, secondary antibodies and streptavidin peroxidase complex reagent successively, and finally the staining was visualized in 3,3'-diaminobenzidine solution.

Slides were blindly scored by two independent pathological investigators unaware of the clinical information, and cases without consensus were evaluated by a third pathologist. The results of IHC were semi-quantified by calculation of the total IHC score, which was the multiplied product of staining score and positive cells score, and the validation cohort was further classified into low-expression group and high-expression group according to the IHC score of detected proteins. The score of staining intensity was defined as: 0 , no staining; 1, weak staining, light yellow; 2, moderate staining, yellowish brown; and 3, strong staining, brown. The score of positively stained tumor cells was defined as: $0,<5 \%$ positive tumor cells; $1,6 \%-30 \%$ positive tumor cells; $2,31 \%-50 \%$ positive tumor cells; and 3 , more than $50 \%$ positive tumor cells. The final score was the multiplied product of the score of staining intensity by the score of positively stained tumor cells, which varied from 0 to 9 . Expression of VEGF-C or NRP2 was identified as: score $\geq 4$ for high expression and score $<4$ for low expression.

\section{Western blotting analysis}

Expression of VEGF-C and NRP2 was detected by Western blotting analysis in U251MG human glioma cells and U118MG and U87MG human glioblastoma cells. Briefly, cells were lysed in the RIPA lysis buffer and centrifuged at $11,000 \mathrm{rpm}$ at $4{ }^{\circ} \mathrm{C}$ for $20 \mathrm{~min}$. Protein concentrations of the supernatants were measured with Bradford kit (Beyotime Institute of Biotechnology, Shanghai, China). Total amount of protein $(10 \mu \mathrm{g})$ was electrophoresed in an SDSPAGE gel, and then transferred to a PVDF membrane (PALL Company, USA). After antigen blocking in 5\% fat-free milk, the membrane with the blotted proteins was incubated with primary antibody overnight at $4{ }^{\circ} \mathrm{C}$. Followed by washing with phosphate-buffered saline for 3 times, the membrane was incubated with secondary antibody labeled with horseradish peroxidase for 2 hours at $37^{\circ} \mathrm{C}$. Finally, the membrane was visualized by ECL substrate (Millipore Company).

\section{Statistical analysis}

All the data were analyzed by software SPSS 17.0. The relationship between the expression of VEGF or NRP2 and clinicopathologic features was analyzed by Chi-square test, and the correlation between the expression of VEGF or NRP2 and the survival rates was evaluated by Kaplan-Meier method, and the difference in survival curves was analyzed by log-rank test. Cox-regression model was used to screen the independent prognostic factor. $\mathrm{P}<0.05$ was considered statistically significant.

\section{Results}

\section{Expression of VEGF-C and NRP2 in glioblastoma tissues} and cell lines

Expression of VEGF-C and NRP2 was detected by IHC. VEGF-C was mainly expressed in cytoplasm (Fig. 1A) and NRP2 expression could be observed in both cytoplasm and membrane (Fig. 1B). Based on the IHC scoring system described in detail in Patients and Materials, the validation cohort were divided into high-expression group and low-expression group (Table 1). The proportion of glioblastoma with high expression of VEGF-C in the entire validation cohort was $34.9 \%$ (30/86), while the proportion 
A

Low-expression

High-expression
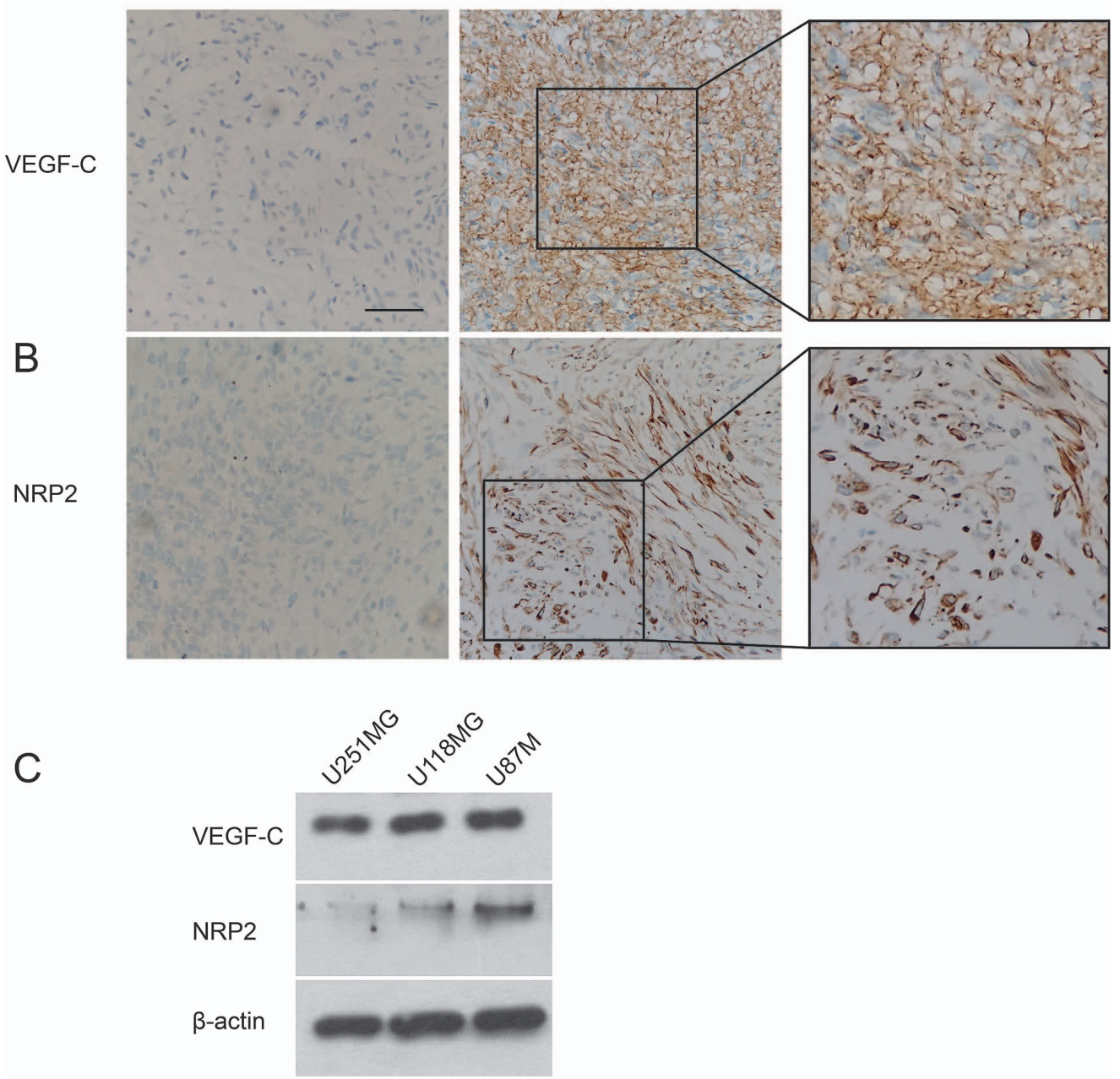

Fig. 1. Representative figures of immunohistochemistry of VEGF-C and NRP2.

A. The representative figures of immunohistochemical staining for low expression (left) and high expression (right) of VEGF-C. Scale bar: $50 \mu \mathrm{m}$.

B. The representative figures of immunohistochemical staining for low expression (left) and high expression (right) of NRP2.

C. Expression of VEGF-C and NRP2 in cell lines U251MG, U118MG and U87MG by immunoblotting analysis.

of glioblastoma with high NRP2 expression was $37.2 \%$ (32/86). To explore the clinical significance of VEGF-C and NRP2 more precisely, we identified the group with concurrent high expression of VEGF-C and NRP2, shown as "VEGF-C\&NRP2" in Table 1, and the remaining cases as control group. In cases with concurrent high expression of VEGF-C and NRP, both VEGF-C and NRP2 were observed as high expression in two parallel separate sections of the same patients. The percentage of "VEGF-C\&NRP2" highexpression group was $15.1 \%(13 / 86)$. Moreover, the proportion of cases with high VEGF-C and low NRP2 was $19.7 \%(17 / 86)$, while the proportion of cases with low VEGF-C and high NRP2 was $22.1 \%$ (19/86).

We then explored the expression of VEGF-C and NRP2 in U251MG glioma cells and U118MG and U87MG glioblastoma cells by immunoblotting analysis (Fig. 1C). It turned out that both VEGF-C and NRP2 were expressed in these three cell lines with different expression abundance. U87MG glioblastoma cells expressed the highest levels of NRP2 and VEGF-C, whereas U251MG glioma cells expressed the lowest NRP2 and VEGF-C levels under the conditions used. These results suggest that cells from more aggressive glioma had higher NRP2 and VEGF-C expression considering that U118MG and U87MG were originated from glioblastoma cells.

Correlations of VEGF-C and NRP2 with other clinicopathologic factors of glioblastoma

The correlation between VEGF-C and/or NRP2 and other clinicopathologic factors was analyzed by Chi-square test to evaluate the clinical significance of VEGF-C and NRP2 (Table 1). The group with low NRP2 expression seemed to have higher probability of complete resection ( $\mathrm{P}$ $=0.038$ ). In addition, male glioblastoma patients were 
Table 1. Correlations between clinicopathologic factors and VEGF-C and/or NRP2.

\begin{tabular}{|c|c|c|c|c|c|c|c|c|c|}
\hline \multirow[b]{2}{*}{ Parameters } & \multicolumn{2}{|c|}{ VEGF-C } & \multirow[b]{2}{*}{$\mathrm{P}^{*}$} & \multicolumn{2}{|c|}{ NRP2 } & \multicolumn{4}{|c|}{ VEGF-C\&NRP2 } \\
\hline & Low & High & & Low & High & $\mathrm{P}^{*}$ & Control $^{\#}$ & High & $\mathrm{P}^{*}$ \\
\hline \multicolumn{10}{|l|}{ Age } \\
\hline$\leq 50$ & 31 & 17 & 0.907 & 29 & 19 & 0.608 & 39 & 9 & 0.283 \\
\hline$>50$ & 25 & 13 & & 25 & 13 & & 34 & 4 & \\
\hline \multicolumn{10}{|l|}{ Gender } \\
\hline Male & 34 & 14 & 0.211 & 24 & 24 & 0.005 & 41 & 7 & 0.877 \\
\hline Female & 22 & 16 & & 30 & 8 & & 32 & 6 & \\
\hline \multicolumn{10}{|l|}{ KPS } \\
\hline$<80$ & 20 & 8 & 0.389 & 20 & 8 & 0.244 & 25 & 3 & 0.417 \\
\hline$\geq 80$ & 36 & 22 & & 34 & 24 & & 48 & 10 & \\
\hline \multicolumn{10}{|c|}{ Extent of resection } \\
\hline Complete & 26 & 18 & 0.229 & 23 & 21 & 0.038 & 36 & 8 & 0.415 \\
\hline Incomplete & 30 & 12 & & 31 & 11 & & 37 & 5 & \\
\hline \multicolumn{10}{|l|}{ VEGF-C } \\
\hline Low & - & - & & 37 & 19 & 0.392 & - & - & \\
\hline High & - & - & & 17 & 13 & & - & - & \\
\hline \multicolumn{10}{|l|}{ NRP2 } \\
\hline Low & 37 & 17 & 0.392 & - & - & & - & - & \\
\hline High & 19 & 13 & & - & - & & - & - & \\
\hline
\end{tabular}

*Calculated by Chi-square test.

\#Control means other groups that include glioblastoma with low expression of VEGF-C and NRP2, glioblastoma with low VEGF-C and high NRP2, and glioblastoma with high VEGF-C and low NRP2.

more likely to have high NRP2 expression $(\mathrm{P}=0.005)$. However, no other significant relations among VEGF-C, NRP2 and clinicopathologic factors were observed.

\section{Concurrent high expression of VEGF and NRP2 correlates to unfavorable prognosis of glioblastoma}

We evaluated the prognostic value of VEGF-C and NRP2 expression by univariate analysis and multivariate analysis. Firstly, the correlations between every clinicopathologic factor and 1-year overall survival rates were analyzed by Kaplan-Merier method (Table 2). Unfortunately, neither VEGF-C nor NRP2 expression could be considered as prognostic factor in univariate analysis $(\mathrm{P}=0.217$ and 0.288 , respectively) (Fig. 2A, B). Considering VEGF-C may function synergistically with NRP2, we explored the prognostic value of high expression of VEGF-C and NRP2. As the result, the group of concurrent high expression of VEGF-C and NRP2 had poorer prognosis than the control group $(\mathrm{P}=0.023)$, indicating that patients with concurrent high expression of VEGF-C and NRP2 may had a more unfavorable outcome, and need chemotherapy more urgently than other patients (Fig. 2C). Moreover, the status of complete/incomplete resection was another prognostic factor $(\mathrm{P}=0.028)$ and patients with incomplete resection had poorer prognosis.

To evaluate whether high expression of VEGF-C and NRP2 was an independent prognostic factor, we performed Cox-regression model and enrolled all available clinico- pathologic parameters for multivariate analysis (Table 3). In the multivariate analysis, high expression of VEGF-C and NRP2 was identified as an independent prognostic factor in glioblastoma $(\mathrm{P}=0.030)$ and no other factors were confirmed.

\section{Discussion}

In glioblastoma, researches of NRP1 function and clinical significance have been got remarkable progresses. NRP1 was considered as a co-receptor for VEGF-induced angiogenesis, and interference of NRP1 could inhibit growth of glioma in vivo (Broholm and Laursen 2004; Nasarre et al. 2010). Moreover, autocrine VEGF-VEGFR2NRP1 signaling was demonstrated to promote glioma stemlike cell viability and tumor growth. However, the exploration of NRP2 effect on glioblastoma is almost vacant compared to the study of NRP1 in glioma, though there were sporadic reports about ectopic expression of NRP2 in other malignancies, including gastric cancer, oral squamous cell carcinomas and bladder cancer (Kim et al. 2009; Keck et al. 2015; Zhang et al. 2015). To the best of our knowledge, we verified the clinical significance of NRP2 in glioblastoma and demonstrated that concurrent high expression of VEGF-C and NRP2 led to poorer prognosis of glioblastoma for the first time. Our study may trigger the interest on VEGF-C and NRP2 study and help find an effective drug target in glioblastoma.

The oncogenic role of VEGF-C and NRP2 has been 
Table 2. Prognostic significance of NRP2 and VEGF-C with univariate analysis.

\begin{tabular}{cccc}
\hline Parameters & mean survival (month) & 1-year survival rate (\%) & P* \\
\hline Age & 13.8 & 52.7 & 0.434 \\
$\leq 50$ & 11.6 & 58.5 & \\
$>50$ & & & 0.308 \\
\hline Gender & 15 & 63.2 & \\
Male & 13.6 & 48.1 & 0.793 \\
Female & & & \\
\hline KPS & 14.1 & 54.4 & 0.028 \\
$<80$ & 13.2 & 55.8 & \\
$\geq 80$ & & 66.9 & 0.217 \\
\hline Extent of resection & 16.2 & 35.9 & \\
Complete & 10.1 & 71.6 & \\
Incomplete & & 38.1 & 0.023 \\
VEGF & 14.6 & & \\
Low & 10 & 50 & \\
High & & 61.3 & \\
\hline NRP2 & 12.5 & 61.3 & \\
Low & 15.5 & & \\
High & 14.7 & & \\
\hline VEGF\&RP2 & 7.5 & & \\
Low & & & \\
High & & & \\
\hline
\end{tabular}

*Calculated by log-rank test.

A
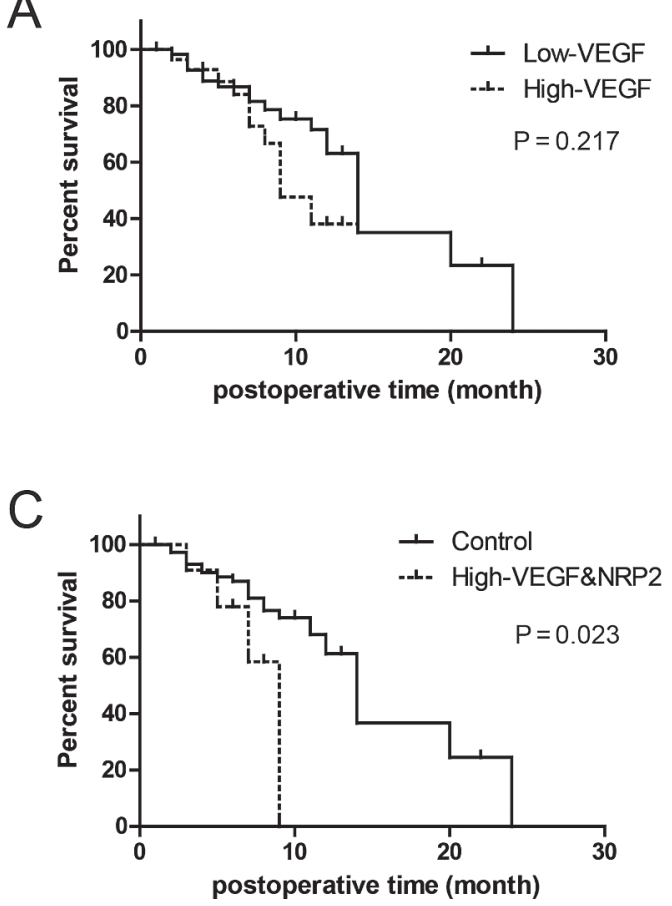

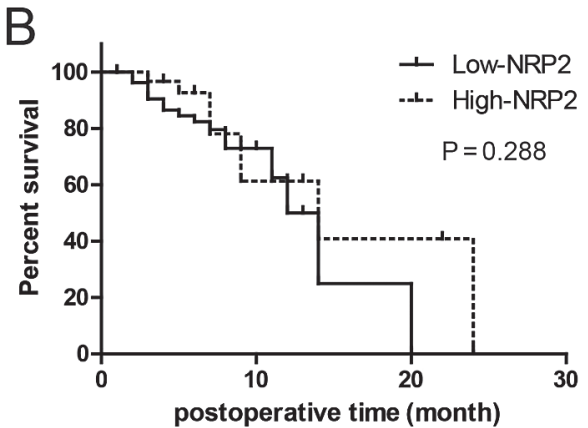

Fig. 2. Prognostic value of VEGF-C and NRP2.

Kaplan-Merrier survival curve was used to display the survival rate. The difference was analyzed by log-rank test between VEGF-C low-expression and high-expression (A), NRP2 low-expression and high-expression (B), and concurrent high expression of VEGF-C and NRP2 and Control (C). 
Table 3. Prognostic value of NRP2 and VEGF-C with multivariate analysis.

\begin{tabular}{cccc}
\hline \multicolumn{4}{c}{ multivariate } \\
Parameters & HR & $95 \%$ CI & $\mathrm{P}^{*}$ \\
\hline Age & & & \\
$\leq 50$ & 1 & & \\
$>50$ & 1.95 & $0.82-4.62$ & 0.129 \\
\hline Gender & 1 & & \\
Male & 0.68 & $0.31-1.50$ & 0.342 \\
Female & & & \\
KPS & 1 & & \\
$<80$ & 1.12 & $0.51-2.48$ & 0.774 \\
$\geq 80$ & & & \\
\hline Extent of resection & 1 & & \\
Complete & 2.79 & $1.21-6.45$ & 0.016 \\
Incomplete & & & \\
\hline VEGF\&RP2 & 1 & & \\
Low & 3.19 & $1.12-9.07$ & 0.03 \\
High & & &
\end{tabular}

*Calculated by Cox-regression model.

revealed in many other kinds of cancers, but they were studied separately instead of considering the prognostic value of concurrent positivity of them. Previous studies proved that VEGF-C could promote the lymphangiogenesis via NRP2 in colorectal carcinoma and oral squamous cell carcinomas et al (Ou et al. 2015; Zhang et al. 2015), and that VEGF-C/NRP2 axis could regulate several biological processes like autophagy besides lymphangiogenesis (Stanton et al. 2013). Moreover, current evidence had revealed the oncogenic role of VEGF-C/NRP2 axis in malignancy such as bladder cancer (Keck et al. 2015). Our finding showed that only concurrent high expression of VEGF-C and NRP2 could influence the prognosis of glioblastoma, which suggested the hypothesis that VEGF-C may stimulate NRP2 and downstream signaling pathway in a paracrine or autocrine pathway in glioblastoma, which is not a rare stimulating mechanism in glioma. This hint indicated a possibility of the clinical usage of antagonist to VEGF-C, NRP2 or other molecular consisting of the VEGF-C/NRP2 secreting or signaling pathway.

It is interesting that NRP2 expression was associated with gender difference in our experiment, suggesting that NRP2 expression may be regulated by the sex hormones. Previous studies have demonstrated that NRP2 mRNA was decreased by estrogen in animal models (Walter et al. 2010), which was consistent with our observation to some extent and may partially elucidate why male patients had higher NRP2 expression in our experiment. However, the underlying mechanisms of the correlation between NRP2 expression and sex hormone level is still misty. Although the prognostic value of VEGF-C/NRP signaling pathway was demonstrated or hinted in several previous studies
(Keck et al. 2015; Kim et al. 2015; Perez et al. 2015; Zhang et al. 2015), the effects of existing VEGF/VEGFR inhibitors such as bevacizumab or sorafenib on glioblastoma treatment are still in clinical trial. We hope our new findings could help identify NRP as a potential drug and help improve the survival time for patients with glioblastoma.

In conclusion, we detected the expression of VEGF-C and NRP2 in human glioblastoma tissues, and proved that double positivity of VEGF-C/NRP2 was significantly associated with the unfavorable prognosis in glioblastoma for the first time. Moreover, concurrent high expression of VEGF-C and NRP2 could be considered as an independent prognostic factor in glioblastoma, indicating that the VEGFC-NRP2 signaling pathway could be a potential and promising drug target in glioblastoma therapy.

\section{Conflict of Interest}

The authors declare no conflict of interest.

\section{References}

Broholm, H. \& Laursen, H. (2004) Vascular endothelial growth factor (VEGF) receptor neuropilin-1's distribution in astrocytic tumors. APMIS, 112, 257-263.

Caunt, M., Mak, J., Liang, W.C., Stawicki, S., Pan, Q., Tong, R.K., Kowalski, J., Ho, C., Reslan, H.B., Ross, J., Berry, L., Kasman, I., Zlot, C., Cheng, Z., Le Couter, J., et al. (2008) Blocking neuropilin-2 function inhibits tumor cell metastasis. Cancer Cell, 13, 331-342.

Epis, M.R., Giles, K.M., Candy, P.A., Webster, R.J. \& Leedman, P.J. (2014) miR-331-3p regulates expression of neuropilin-2 in glioblastoma. J. Neurooncol., 116, 67-75.

Goel, H.L., Chang, C., Pursell, B., Leav, I., Lyle, S., Xi, H.S., Hsieh, C.C., Adisetiyo, H., Roy-Burman, P., Coleman, I.M., Nelson, P.S., Vessella, R.L., Davis, R.J., Plymate, S.R. \& Mercurio, A.M. (2012) VEGF/neuropilin-2 regulation of Bmi-1 and consequent repression of IGF-IR define a novel mechanism of aggressive prostate cancer. Cancer Discov., 2, 906-921.

Hamerlik, P., Lathia, J.D., Rasmussen, R., Wu, Q., Bartkova, J., Lee, M., Moudry, P., Bartek, J. Jr., Fischer, W., Lukas, J., Rich, J.N. \& Bartek, J. (2012) Autocrine VEGF-VEGFR2-Neuropilin-1 signaling promotes glioma stem-like cell viability and tumor growth. J. Exp. Med., 209, 507-520.

Keck, B., Wach, S., Taubert, H., Zeiler, S., Ott, O.J., Kunath, F., Hartmann, A., Bertz, S., Weiss, C., Honscheid, P., Schellenburg, S., Rodel, C., Baretton, G.B., Sauer, R., Fietkau, R., et al. (2015) Neuropilin-2 and its ligand VEGF-C predict treatment response after transurethral resection and radiochemotherapy in bladder cancer patients. Int. J. Cancer, 136, 443-451.

Kim, H.Y., Rha, K.S., Shim, G.A., Kim, J.H., Kim, J.M., Huang, S.M. \& Koo, B.S. (2015) Podoplanin is involved in the prognosis of head and neck squamous cell carcinoma through interaction with VEGF-C. Oncol. Rep., 34, 833-842.

Kim, W.H., Lee, S.H., Jung, M.H., Seo, J.H., Kim, J., Kim, M.A. \& Lee, Y.M. (2009) Neuropilin2 expressed in gastric cancer endothelial cells increases the proliferation and migration of endothelial cells in response to VEGF. Exp. Cell Res., 315, 2154-2164.

Li, X., Tang, T., Lu, X., Zhou, H. \& Huang, Y. (2011) RNA interference targeting NRP-1 inhibits human glioma cell proliferation and enhances cell apoptosis. Mol. Med. Rep., 4, 12611266

Louis, D.N., Ohgaki, H., Wiestler, O.D., Cavenee, W.K., Burger, P.C., Jouvet, A., Scheithauer, B.W. \& Kleihues, P. (2007) The 
2007 WHO classification of tumours of the central nervous system. Acta Neuropathol., 114, 97-109.

Meng, D., Chen, Y., Yun, D., Zhao, Y., Wang, J., Xu, T., Li, X., Wang, Y., Yuan, L., Sun, R., Song, X., Huai, C., Hu, L., Yang, S., Min, T., et al. (2015) High expression of N-myc (and STAT) interactor predicts poor prognosis and promotes tumor growth in human glioblastoma. Oncotarget, 6, 4901-4919.

Nasarre, C., Roth, M., Jacob, L., Roth, L., Koncina, E., Thien, A., Labourdette, G., Poulet, P., Hubert, P., Cremel, G., Roussel, G., Aunis, D. \& Bagnard, D. (2010) Peptide-based interference of the transmembrane domain of neuropilin-1 inhibits glioma growth in vivo. Oncogene, 29, 2381-2392.

Nicolaidis, S. (2015) Biomarkers of glioblastoma multiforme. Metabolism, 64, S22-S27.

Ou, J.J., Wei, X., Peng, Y., Zha, L., Zhou, R.B., Shi, H., Zhou, Q. \& Liang, H.J. (2015) Neuropilin-2 mediates lymphangiogenesis of colorectal carcinoma via a VEGFC/VEGFR3 independent signaling. Cancer Lett., 358, 200-209.

Perez, D., Rohde, A., Callejon, G., Perez-Ruiz, E., Rodrigo, I., Rivas-Ruiz, F., Ramos, B., Medina, F., Villatoro, R., Redondo, M., Zarcos, I., Maanon, C. \& Rueda, A. (2015) Correlation between serum levels of vascular endothelial growth factor-C and sentinel lymph node status in early breast cancer. Tumour Biol., 36, 9285-9293.

Plein, A., Fantin, A. \& Ruhrberg, C. (2014) Neuropilin regulation of angiogenesis, arteriogenesis, and vascular permeability. Microcirculation, 21, 315-323.
Prud'homme, G.J. \& Glinka, Y. (2012) Neuropilins are multifunctional coreceptors involved in tumor initiation, growth, metastasis and immunity. Oncotarget, 3, 921-939.

Stanton, M.J., Dutta, S., Zhang, H., Polavaram, N.S., Leontovich, A.A., Honscheid, P., Sinicrope, F.A., Tindall, D.J., Muders, M.H. \& Datta, K. (2013) Autophagy control by the VEGF-C/ NRP-2 axis in cancer and its implication for treatment resistance. Cancer Res., 73, 160-171.

Sulpice, E., Plouet, J., Berge, M., Allanic, D., Tobelem, G. \& Merkulova-Rainon, T. (2008) Neuropilin-1 and neuropilin-2 act as coreceptors, potentiating proangiogenic activity. Blood, 111, 2036-2045.

Walter, L.M., Rogers, P.A. \& Girling, J.E. (2010) Vascular endothelial growth factor-A isoform and (co)receptor expression are differentially regulated by 17 beta-oestradiol in the ovariectomised mouse uterus. Reproduction, 140, 331-341.

Wen, P.Y. \& Kesari, S. (2008) Malignant gliomas in adults. $N$. Engl. J. Med., 359, 492-507.

Wrensch, M., Minn, Y., Chew, T., Bondy, M. \& Berger, M.S. (2002) Epidemiology of primary brain tumors: current concepts and review of the literature. Neuro. Oncol., 4, 278-299.

Zhang, B., Gao, Z., Sun, M., Li, H., Fan, H., Chen, D. \& Zheng, J. (2015) Prognostic significance of VEGF-C, semaphorin 3F, and neuropilin-2 expression in oral squamous cell carcinomas and their relationship with lymphangiogenesis. J. Surg. Oncol., 111, 382-388. 